\title{
Experimental feasibility of spectral photon-counting computed tomography with two contrast agents for the detection of endoleaks following endovascular aortic repair
}

\author{
Julia Dangelmaier $^{1}$ (1) - Daniel Bar-Ness ${ }^{2} \cdot$ Heiner Daerr $^{3} \cdot$ Daniela Muenzel $^{1} \cdot$ Salim Si-Mohamed ${ }^{2,4,5} \cdot$ Sebastian Ehn $^{6}$. \\ Alexander A. Fingerle ${ }^{1}$ - Melanie A. Kimm ${ }^{1}$ • Felix K. Kopp ${ }^{1}$ • Loic Boussel $^{2,4,5}$ • Ewald Roessl ${ }^{3}$ - Franz Pfeiffer B $^{1,6}$. \\ Ernst J. Rummeny ${ }^{1} \cdot$ Roland Proksa $^{3}$ • Philippe Douek ${ }^{2,4,5} \cdot$ Peter B. Noël $^{1,6}$
}

Received: 9 October 2017 / Revised: 30 November 2017 / Accepted: 8 December 2017 / Published online: 19 February 2018

(C) The Author(s) 2018. This article is an open access publication

\begin{abstract}
Objectives After endovascular aortic repair (EVAR), discrimination of endoleaks and intra-aneurysmatic calcifications within the aneurysm often requires multiphase computed tomography (CT). Spectral photon-counting CT (SPCCT) in combination with a two-contrast agent injection protocol may provide reliable detection of endoleaks with a single CT acquisition.

Methods To evaluate the feasibility of SPCCT, the stent-lined compartment of an abdominal aortic aneurysm phantom was filled with a mixture of iodine and gadolinium mimicking enhanced blood. To represent endoleaks of different flow rates, the adjacent compartments contained either one of the contrast agents or calcium chloride to mimic intra-aneurysmatic calcifications. After data acquisition with a SPCCT prototype scanner with multi-energy bins, material decomposition was performed to generate iodine, gadolinium and calcium maps.

Results In a conventional CT slice, Hounsfield units (HU) of the compartments were similar ranging from 147 to $168 \mathrm{HU}$. Material-specific maps differentiate the distributions within the compartments filled with iodine, gadolinium or calcium.

Conclusion SPCCT may replace multiphase CT to detect endoleaks without sacrificing diagnostic accuracy. It is a unique feature of our method to capture endoleak dynamics and allow reliable distinction from intra-aneurysmatic calcifications in a single scan, thereby enabling a significant reduction of radiation exposure.

Key Points

- SPCCT might enable advanced endoleak detection.

- Material maps derived from SPCCT can differentiate iodine, gadolinium and calcium.

- SPCCT may potentially reduce radiation burden for EVAR patients under post-interventional surveillance.
\end{abstract}

Keywords Computed tomography $\cdot$ Photon counting $\cdot$ Endoleak $\cdot$ Iodine $\cdot$ Gadolinium

Julia Dangelmaier

julia.dangelmaier@tum.de

1 Department of Diagnostic and Interventional Radiology, Technische Universität München, Ismaningerstr. 22, 81675 Munich, Germany

2 CREATIS, CNRS UMR 5220, INSERM U1206, INSA, Lyon, France

3 Philips GmbH Innovative Technologies, Research Laboratories, Hamburg, Germany

4 Radiology Department, Lyon University Hospital, Lyon, France

5 University Lyon1 Claude Bernard, Lyon, France

6 Chair of Biomedical Physics, Department of Physics and Munich School of BioEngineering, Technische Universität München, 85748 Garching, Germany

\author{
Abbreviations \\ AAA Abdominal aortic aneurysm \\ ASIC Application-specific integrated circuit \\ CT Computed tomography \\ CZT Cadmium zinc telluride \\ DECT Dual-energy computed tomography \\ EVAR Endovascular aortic repair \\ HU Hounsfield units \\ MD Material decomposition \\ SNR Signal-to-noise ratio \\ SPCCT Spectral photon-counting computed tomography
}




\section{Introduction}

An abdominal aortic diameter of $3 \mathrm{~cm}$ or more is considered as an abdominal aortic aneurysm (AAA). AAAs have a prevalence of $1.6-7.2 \%[1,2]$ and an incidence of $0.4-0.7 \%$ per year in the Western population [3]. Symptomatic aneurysms (e.g. abdominal pain) and rapid growing aneurysms (more than $5 \mathrm{~mm}$ in 6 months) [4] as well as AAAs measuring greater than $5.5 \mathrm{~cm}$ at baseline are in need to be treated [5]. Next to open surgery, minimal invasive implantation of a covered, self-expandable stent graft - the endovascular aortic repair (EVAR) - is well established since it was first presented by Parodi et al. in 1991 [6]. An endoleak is the most frequent complication (53\% of all complications; incidence of $11.7 \%$ ) following EVAR [7] and typically requires a secondary intervention [8], since it promotes further growing of the aneurysm (in $41 \%$ of patients), which can disastrously lead to aortic rupture (in 2.4\%) [7].

The Society for Vascular Surgery recommends life-long follow-ups (1, 6 and 12 months after the intervention and annually thereafter) using contrast-enhanced computed tomography (CT) scans to detect possible complications related to EVAR [9]. Because of different flow rates, endoleaks can manifest in the arterial (high flow) and/or in the venous or delayed phase (60-120 s post injection) (low flow) and might be difficult to distinguish from intra-aneurysmatic calcifications (Fig. 1). Therefore, standard CT protocols include a native scan of the abdomen (or just the stent graft area recognized by the survey scan), a scan of the whole abdomen in the arterial phase after intravenous contrast agent application and a delayed scan of the whole abdomen or stent graft area [10, 11]. A CT scan in the arterial phase also enables the evaluation of the access vessels and an in-stent lumen visualization, whereby organ infarction - a potential adverse effect of vascular intervention - is usually reliably detectable in the delayed phase scan.

However, repetitive multiphasic CT scans lead to a critical accumulative radiation dose. Dual-energy CT (DECT) allows decomposition into two basis materials and offers the possibility to reduce radiation burden in comparison to multiphase studies e.g. by providing a virtual non-contrast image [12]. The introduction of the current preclinical technique of spectral photon-counting CT (SPCCT) [13-17] could even further reduce radiation dose for patients under surveillance following EVAR. Spectral photon-counting detectors employed in SPCCT count incoming photons, bin them with respect to their energy and are therefore able to discriminate more than two different materials [18-20]. In addition, SPCCT is capable of ultra-high resolution imaging, improved signal-to-noise (SNR) and reduction of beam hardening artefacts.

Surveillance of EVAR patients with SPCCT will require a dedicated injection protocol for intravenous application of two contrast agents (e.g. gadolinium and iodine) (Fig. 2). A region of interest (ROI) for automated detection of contrast enhancement should be placed within the lumen of the stent-graft, and peak enhancement by gadolinium, injected at $\mathrm{T} 0$, should be registered (T1). Hence, the period T1-T0 defines the time necessary for a maximal enhancement in the arterial phase and it is an individual parameter that varies from patient to patient in relation to, among others, their cardiac output and aortic diameter. While the attenuation by gadolinium within the lumen of the stent-graft will decrease, the attenuation within the aneurysm sac will increase because of the endoleak. Under consideration of T1-T0 iodine should be injected (T2) in order to meet a venous/delayed phase $(60-120 \mathrm{~s}$ post injection) for the initially applied gadolinium and an arterial distribution of iodine, when a single SPCCT scan is performed at $\mathrm{T} 3=\mathrm{T} 2+\mathrm{T} 1-\mathrm{T} 0$.

In this study, we demonstrate in a phantom model that a single SPCCT scan could capture endoleak dynamics and discriminate endoleaks from intra-aneurysmatic calcifications.

\section{Material and methods}

\section{Aortic aneurysm phantom}

An aortic aneurysm phantom was designed in-house and produced by a selective laser sintering of polyamide. The phantom was based on a conventional CT scan obtained in clinical routine following EVAR and planned in due consideration of our injection protocol (Fig. 2). The phantom contains eight compartments of different sizes and configurations arranged as tubes on a closed base. A centrally located compartment is lined up with a $36 \mathrm{~mm} \times 100 \mathrm{~mm}$ covered stent-graft (Endurant, Medtronic, Germany) and filled with distilled water, iodine (Solutrast 370, Bracco, Germany; $370 \mathrm{mg}$ iodine/ $\mathrm{ml}$ ) and gadolinium (Magnograf $0.5 \mathrm{mmol} / \mathrm{ml}$, Jenapharm, Germany; $78.63 \mathrm{mg}$ gadolinium $/ \mathrm{ml}$ ) mimicking enhanced blood within the stent lumen. Immediately adjacent to it, there is a compartment containing iodine (6 mg iodine $/ \mathrm{ml}$; Solutrast diluted in distilled water) that represents the leaking contrast media in the arterial phase injected at T2. Surrounding these, a gadolinium-filled compartment represents leaking in the venous/delayed phase ( $4 \mathrm{mg}$ gadolinium $/ \mathrm{ml}$; Magnograf diluted in distilled water). Interposed calcium chloride ( $75 \mathrm{mg}$ calcium chloride/ml; diluted in distilled water)-filled compartments mimic intra-aneurysmatic calcifications. Compartment 5 represents the excluded aneurysm sac and was filled with an oral contrast media used regularly in magnetic resonance imaging (Lumivision, Bender, Germany) to mimic clotted blood. Lumivision provides Hounsfield unit (HU) values ranging from 40 to $75 \mathrm{HU}$, which are frequently clinically measured within the excluded aneurysm sac following EVAR.

Likewise, the intensities of all components were based on $\mathrm{HU}$ measured in a routinely obtained aortic CT scan. Solutrast, 
Fig. 1 An 82-year-old EVAR patient $(\mathrm{m})$ undergoing follow-up with a triphasic CT scan (level $100 \mathrm{HU}$, window $600 \mathrm{HU}) 1$ year after intervention. The arterial phase (a) and venous phase (b) show a low flow endoleak (arrowhead) apparent only in the venous phase (b). The native scan (c) identifies hyperdense material within the aneurysm sac as calcifications (arrows). These could be mistaken as an endoleak in the contrast-enhanced scan (d)

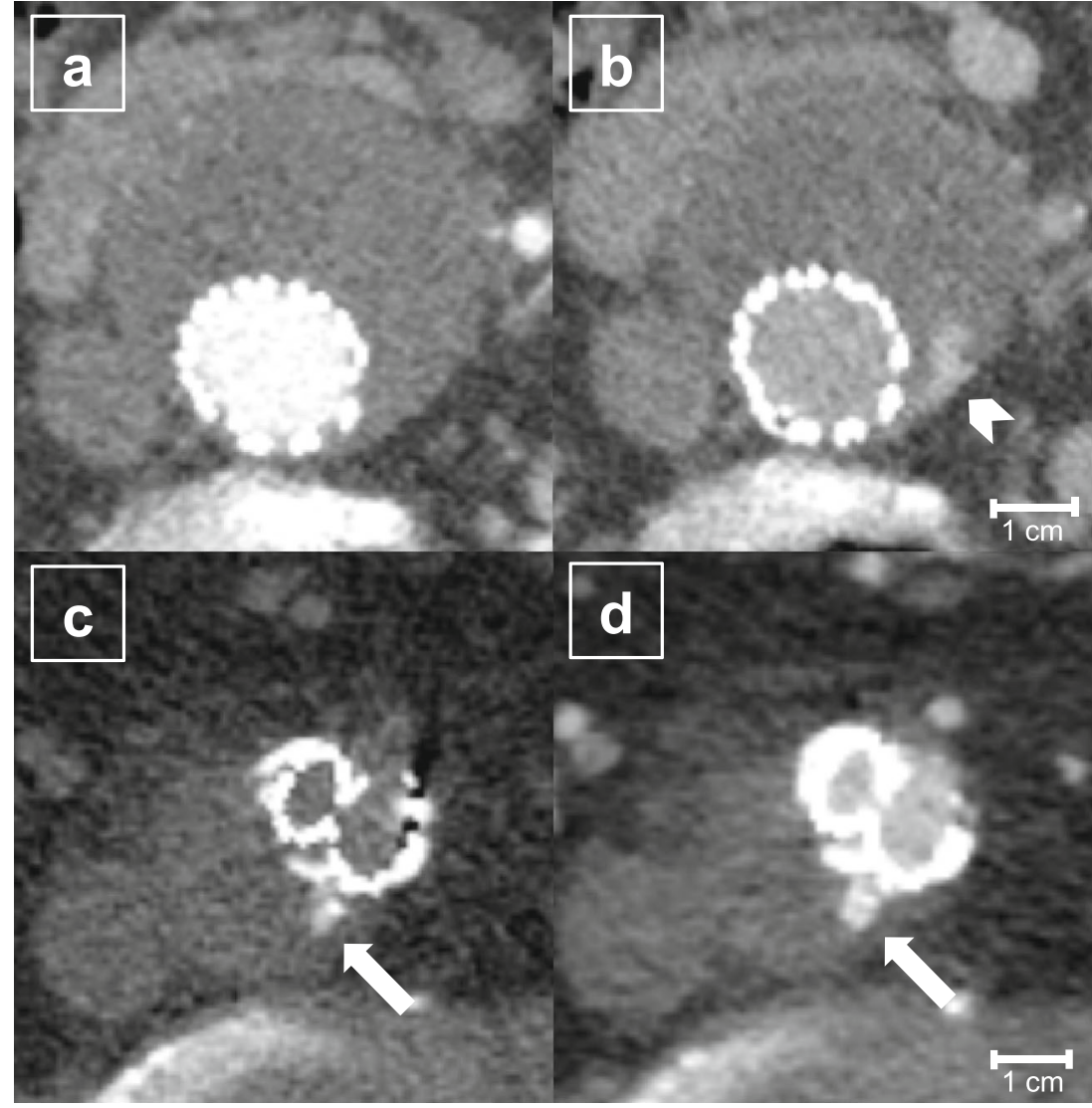

Magnograf and calcium chloride were diluted in distilled water until similar intensities (approx. $150 \mathrm{HU}$ at a tube voltage of $120 \mathrm{kVp}$ ) were reached. Solutions were shaken immediately prior to the SPCCT scan to avoid sedimentation.

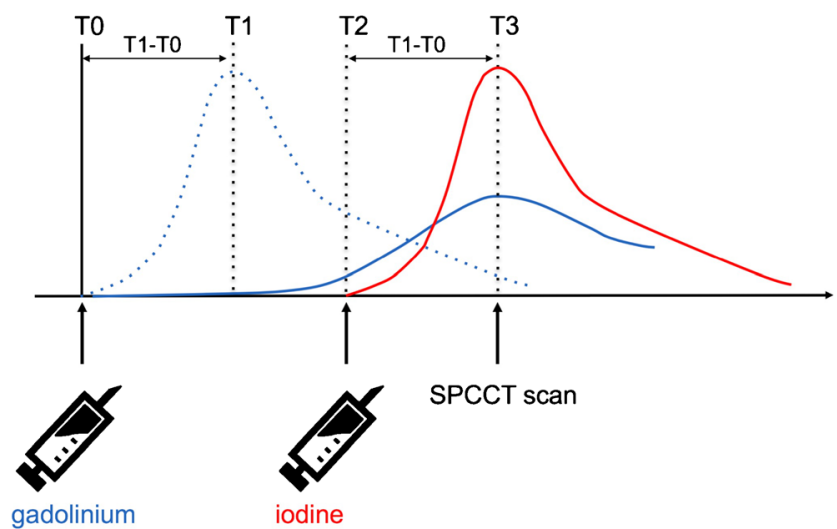

Fig. 2 Dual contrast agent injection protocol for endoleak detection by a single SPCCT scan. Peak enhancement by gadolinium, injected at T0, should be registered (T1) within the lumen of the stent-graft. The time period $\mathrm{T} 1-\mathrm{T} 0$ defines the time necessary for maximal enhancement in the arterial phase. Under consideration of T1-T0, iodine should be injected (T2) in order to meet a venous/delayed distribution for the initially applied gadolinium and an arterial distribution of iodine, when a single SPCCT scan is performed at $\mathrm{T} 3=\mathrm{T} 2+\mathrm{T} 1-\mathrm{T} 0$. (Blue dotted line, arterial distribution of gadolinium; solid blue line, venous/delayed distribution of gadolinium; red line, arterial distribution of iodine)

\section{Spectral photon-counting CT examination}

The phantom was scanned with a multi-bin preclinical SPCCT system (Philips Healthcare, Haifa, Israel) using a step-and-shoot acquisition mode with an X-ray tube current of $100 \mathrm{~mA}$, an X-ray tube voltage of $120 \mathrm{kVp}$ and a scanner rotation time of $1 \mathrm{~s}$. The scanner is based on a clinical CT system (Brilliance iCT, Philips Healthcare, Haifa, Israel) providing a conventional $\mathrm{X}$-ray tube and standard beam filtration but with a limited in-plane field of view of $168 \mathrm{~mm}$ and a zcoverage of $2.5 \mathrm{~mm}$ at isocentre. The hybrid photon-counting detectors ChromAIX2 ASICs (application-specific integrated circuit) [21] combined with cadmium zinc telluride (CZT) as sensor material have the capability to set five energy threshold values. The threshold positions were optimized to maximize the image signal-to-noise ratio [22]. They were set to 30,51 , 64, 72 and $85 \mathrm{keV}$ in order to achieve high sensitivity for the different materials scanned, including gadolinium, which has a K-edge at $50.24 \mathrm{keV}$.

\section{Material decomposition, processing and image reconstruction}

A conventional image data set was reconstructed from preprocessed photon counts of all energy bins using filtered back-projection. The processing included a metal artefact 
reduction. Apart from this, two binary material maps were generated comprising the information whether an image voxel of the conventional image is more likely to contain iodine or calcium. To obtain the information about the material distributions within the phantom, the pre-processed photon counts were decomposed into three projection data sets of photoelectric effect, Compton effect and gadolinium by a maximumlikelihood approach [23]. The projections were separately reconstructed to images individually again by conventional filtered back-projection. For iodine and calcium, probability distributions were calculated from the images of the photoelectric effect and the Compton effect. The X-ray attenuation of different materials without K-edge absorption in the selected energy range can be described by a varying amount of attenuation by the photoelectric effect and the Compton effect [24] as illustrated in Fig. 3a. By evaluating for each image voxel the values in the photoelectric and Compton images, an image-based material separation of iodine and calcium was possible. The distributions of iodine and calcium both cluster around different mean value and were modelled by joint real normal distributions (Fig. 3b). By evaluating the distribution models for each image voxel probability maps for iodine and calcium were calculated. The images were statistically filtered taking into account the anti-correlated noise to reduce the overlap of the formed clusters [25]. For each image voxel, a binary decision was made whether the voxel contained calcium or iodine on the basis of the probability maps.

\section{Data analysis}

The iodine and gadolinium concentrations were determined experimentally by material decomposition and by conversion of the $\mathrm{CT}$ values in the conventional image to concentrations via a look-up table. CT values of iodine and gadolinium and their concentrations obtained from the material-specific maps were measured by a single observer. Regions of interest (ROIs) where drawn with identical size and position in the conventional CT images as well as in the material maps. To demonstrate the visually improved differentiation between the different compartments a line profile representing the values in conventional and material maps was drawn. For improved comparability, the different images were scaled between zero and one for visualization of the line profiles.

\section{Results}

Figure 4 shows a photographic image (a) as well as axial tomographic images $(\mathrm{b}-\mathrm{f})$ of the aortic phantom. Materialspecific maps were created as an overlay of all material maps (c) and separately for calcium (d), gadolinium (e) and iodine (f). Measured in the conventional CT (Fig. 4b), the intensity of the ROIs placed in different compartments $1-5$ were $151 \mathrm{HU}$,
$147 \mathrm{HU}, 168 \mathrm{HU}, 340 \mathrm{HU}$ and $40 \mathrm{HU}$ with a standard deviation of $8 \mathrm{HU}$ at $3 \mathrm{~mm}$ slice width, respectively. Of note, the iodine, gadolinium and calcium compartments were indistinguishable in Fig. 4b, illustrating a frequently occurring problem in clinical routine. On the SPCCT data, areas of the phantom containing gadolinium could be clearly identified on the basis of their characteristic increase of attenuation at the $\mathrm{K}$ edge. Iodine and calcium probability maps_-based on photoelectric effect and Compton effect - showed a slight overlap at given material concentrations and applied dose. However, both compartments were clearly assignable within the phantom.

The estimated concentration of iodine based on the CT value of $147 \pm 3 \mathrm{HU}$ was $4.9 \pm 0.1 \mathrm{mg} / \mathrm{ml}$ and the estimated concentration of gadolinium based on $168 \pm 4 \mathrm{HU}$ was $3.5 \pm$ $0.1 \mathrm{mg} / \mathrm{ml}$. Using material decomposition, we measured the concentration of iodine as $3.6 \pm 0.2 \mathrm{mg} / \mathrm{ml}$ and the concentration of gadolinium as $3.2 \pm 0.1 \mathrm{mg} / \mathrm{ml}$. Comparable errors of $1.2 \mathrm{mg} / \mathrm{ml}$ are also reported and discussed by Cormode et al. [17].

Figure 5 illustrates the improved differentiability when comparing conventional $\mathrm{CT}$ versus material-specific maps. The paths through the compartments filled with iodine, gadolinium and calcium are indicated with red and green lines in Fig. 5a. Figure 5b shows that with similar HU values in the conventional CT, the differentiation is impossible; whereas, it is possible with the material-specific maps. For improved comparability, the different images were scaled between zero and one for visualization of the line profiles.

\section{Discussion}

This study provides first proof-of-concept results for SPCCT combined with a dedicated dual contrast agent application for reliable endoleak detection with a single CT acquisition, potentially lowering radiation dose for patients under surveillance following EVAR.

Since intra-aneurysmatic calcifications might be difficult to distinguish from leaking contrast media and endoleaks possess different flow rates, triphasic CT protocols are indispensable but add up to a significant radiation burden for the patient. Kalef-Ezra et al. point out an effective dose of $62 \mathrm{mSv}$ only in the first year following EVAR [26]. In 2013 Brägelmann et al. reported a cumulative dose of $18.67 \mathrm{mSv}$ for a triphasic CT protocol, consisting of a native $(4.53 \mathrm{mSv})$, an arterial (7.13 $\mathrm{mSv})$ and a delayed phase (7.01 mSv) scan [12]. Brägelmann et al. were able to show a dose reduction to $14.14 \mathrm{mSv}$ by replacing the true native scan with a virtual native scan using a DECT scanner. Furthermore, the acquisition of only one single DECT scan in the delayed phase enabled an effective dose reduction of $62 \%$, but can only provide limited information about the arterial flow path. Recently published work 
Fig. 3 Image-based separation of calcium and iodine: distribution of photoelectric effect and Compton effect image (a) and modelling of material distributions of iodine and calcium (b)

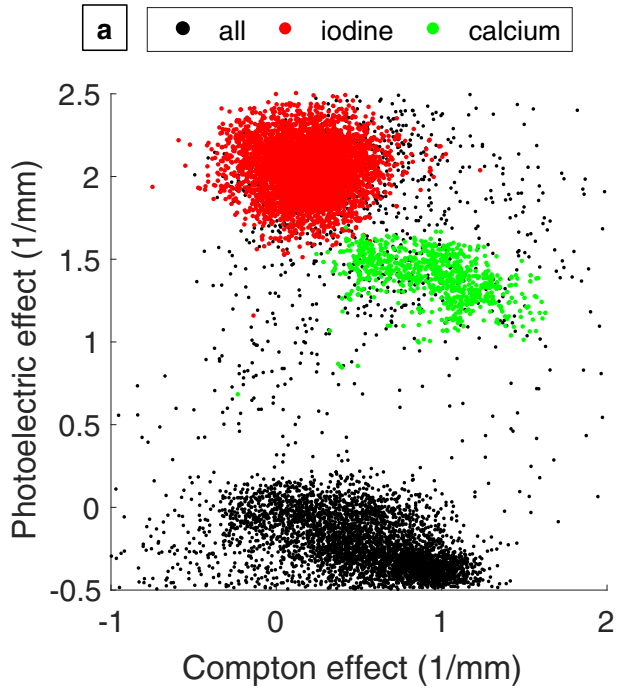

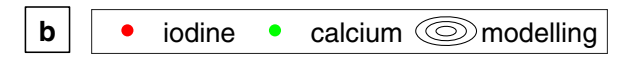

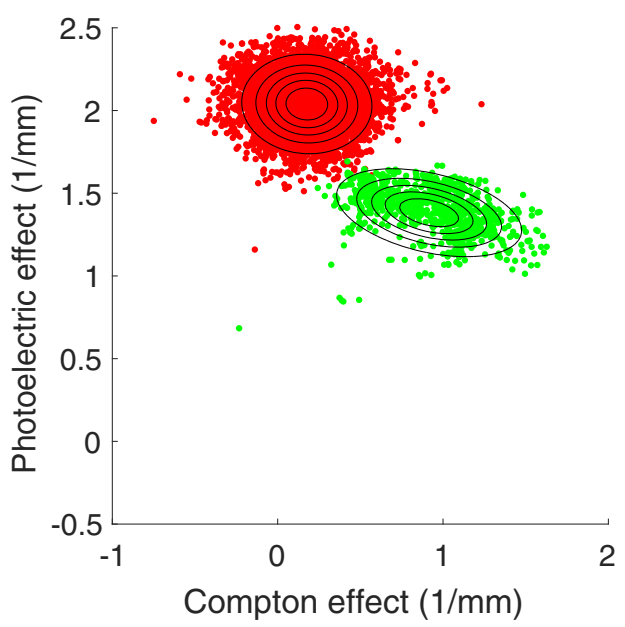

proposes a single-acquisition split-bolus DECT [27] providing the possibility to capture biphasic information within a single CT scan. Javor et al.'s analysis showed a significant reduction of radiation burden of up to $42 \%$, while maintaining a sufficient diagnostic quality with an endoleak detection rate of $96 \%$ [27]. Unfortunately, the type of endoleak (either low or high flow) cannot be precisely determined, since with mixed arterio-venous contrast leakage is not assignable to the arterial or venous phase.

With the introduction of SPCCT, the possibility arises to answer clinically relevant questions by distinguishing two contrast agents. The important advantages of a SPCCT, compared to current CT technology, are based on the concept that incoming photons are counted with respect to their energy within predefined energy windows [18-20]. This technology offers the possibility to perform multi-material decomposition including K-edge imaging. Initial pilot studies have demonstrated the possibility to employ SPCCT data for diagnostic tasks [14-16, 21, 28-30]. Muenzel et al. demonstrated the feasibility of SPCCT-colonography to differentiate iodinetagged faeces or fluids and gadolinium-enhanced polyps. Material maps clearly differentiated the distributions of gadolinium and iodine, and quantitative measurements of the material concentrations could also be performed with high accuracy [15]. Symons et al. successfully performed the decomposition of three contrast agents in a large animal model SPCCT scan. Distribution of intravenous successively administered gadolinium and iodine and oral applied bismuth,
Fig. 4 Photograph of the aortic phantom (a), conventional CT scan (b), overlay of the three material maps $(\mathbf{c})($ green $=$ calcium; red = iodine; blue = gadolinium), calcium map (d), gadolinium map (e) and iodine map (f) (level - $153 \mathrm{HU}$, window $1120 \mathrm{HU})$.

1 = calcium; 2 = gadolinium; 3 = iodine; 4 = stent lined, iodine and gadolinium. 5 = oral contrast media

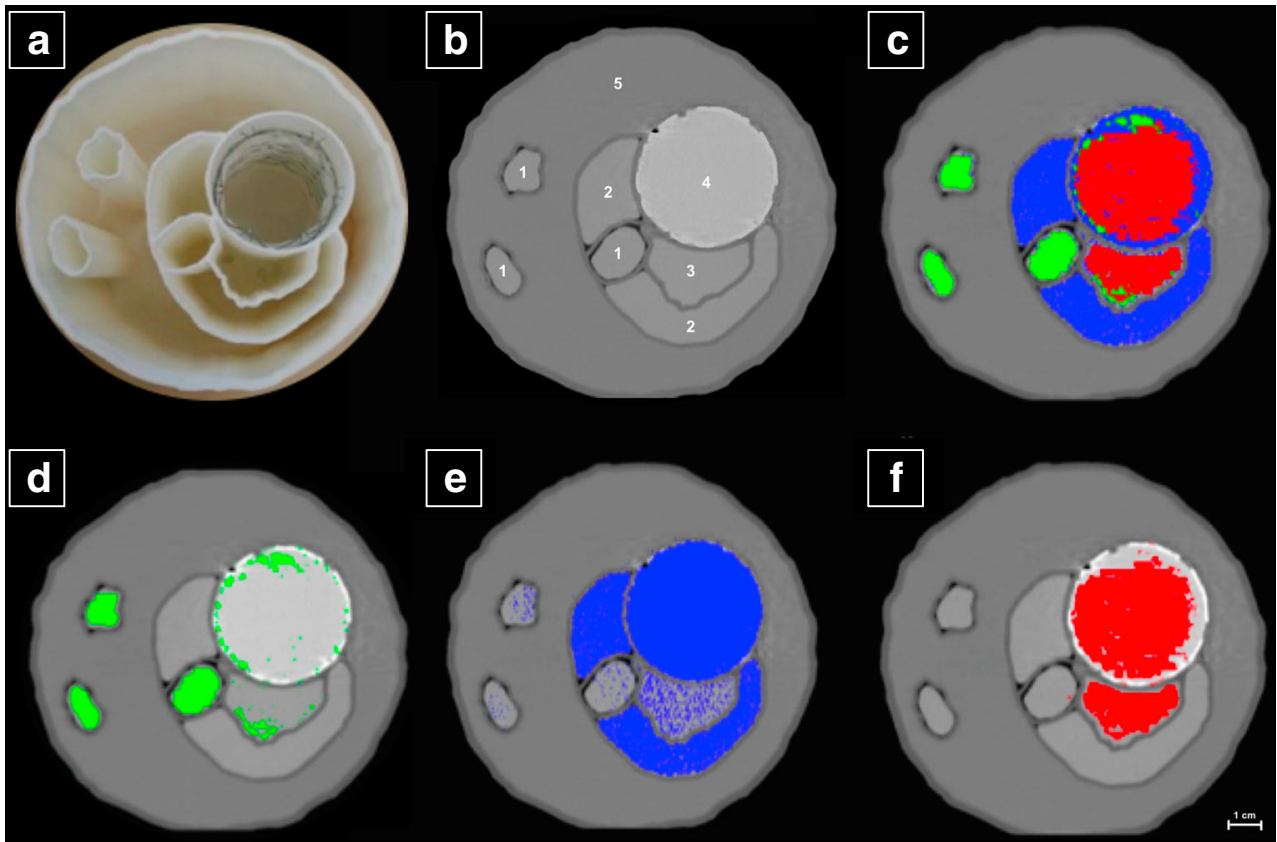


Fig. 5 a Normalized conventional greyscale image of the aortic aneurysm phantom obtained with SPCCT. b Line plots, indicated in a with red and green lines, are tracking through the compartments filled with iodine, gadolinium and calcium. Intensities measured in the conventional SPCCT image (blue line), measured in the gadolinium map (orange line), obtained from the iodine probability map (grey line) and the calcium probability map (yellow line) are displayed
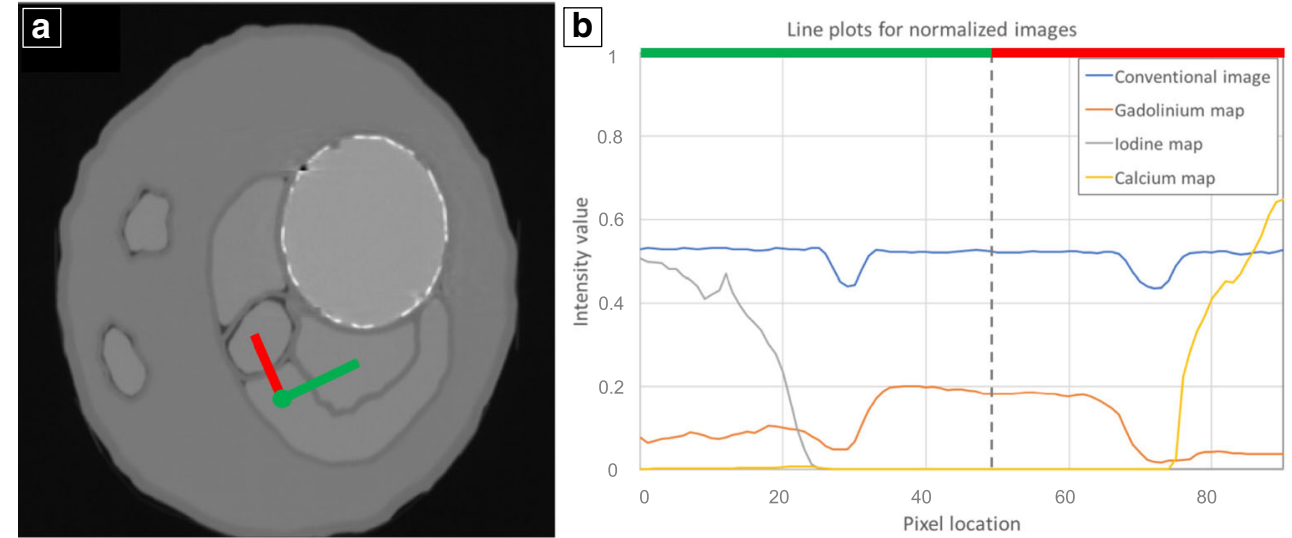

indistinguishable on the grayscale images, could be clearly differentiated with the SPCCT images. Symons et al. propose that, split-bolus dual-contrast SPCCT for multiphase kidney imaging could reduce radiation burden in comparison to conventional multiphase $\mathrm{CT}$ imaging [28].

Along the same lines, a single SPCCT scan in combination with a successive application of intravenous applied contrast media (e.g. iodine and gadolinium) can provide spatial and temporal information to detect endoleaks following EVAR. It allows observation of flow dynamics as well as the reliable distinction of leaking contrast media and intra-aneurysmatic calcifications, since identification of more than two materials is enabled. With respect to the diagnostic image quality, an improvement of spatial resolution, SNR and beam-hardening artefacts reduction can be expected. Additional misalignment of conventional CT scans in native, arterial and venous phase due to movement or breathing by the patient will be avoided. Consequently, by applying just one scan with an X-ray dose comparable to a single scan, SPCCT could significantly reduce the radiation burden for patients under surveillance following EVAR without sacrificing the diagnostic accuracy or may even improve it.

Presently, simultaneous applications of the two contrast agents iodine and gadolinium is not approved and needs pharmacological evaluation before the proposed injection protocol can be applied for patient care. Furthermore, current finding of depletions of specific (e.g. linear) gadolinium contrast agent in the dentate nucleus and pallidum after repetitive application, even in patients without renal impairment [31], questions the utilization of gadolinium-containing contrast media for novel clinical applications. However, the suggested injection protocol could be universally usable for various contrast media. Our injection protocol even provides the possibility to minimize the amount of applied contrast agent at the time point $\mathrm{T} 2$, because the triggering of the initially applied contrast agent allows reliable assessment of optimal enhancement and scan time. Therefore, dosage of contrast agent applied at T2 can be adjusted and potential side effects probably reduced.
The minimum of detectable leaking contrast media and minimal amount of discriminable contrast media towards intra-aneurysmatic calcifications cannot be assessed in the context of our experimental set-up. Calcium and iodine maps showed a slight overlap and this effect has to be further evaluated and revealed in vivo. Utilization of two K-edge materials would allow an easier and more reliable discrimination towards calcifications with high SNR.

With regard to the possibilities of SPCCT, K-edge imaging-compatible contrast agents would be of particular interest. Unfortunately, clinically approved iodine-based contrast agents have a relatively low $\mathrm{K}$-edge $(33.17 \mathrm{keV})$, which is unfeasible for $\mathrm{K}$-edge imaging in clinical routine because of absorption of low energy photons below the K-edge. Therefore, the development and further promotion of new contrast agents, which are partially on the product roadmap [32], is of particular interest to fully exploit the benefits of SPCCT imaging.

\section{Conclusion}

SPCCT in combination with a dual contrast agent injection protocol may replace conventional CT scans in native, arterial and delayed phase to detect endoleaks without sacrificing the diagnostic accuracy. It is a unique feature of our method to capture endoleak dynamics and allow distinction from intraaneurysmatic calcifications in a single scan. This novel approach will potentially realize a relevant reduction of the radiation burden for patients under CT surveillance following EVAR.

Funding This study has received funding by the European Research Council (ERC, H2020, AdG 695045), the DFG Gottfried Wilhelm Leibniz program, by the German Department of Education and Research (BMBF) under grant IMEDO (13GW0072C), the support of the TUM Institute for Advanced Study, funded by the German Excellence Initiative and European Union's Horizon 2020 research and innovation programme under grant agreement No 633937 


\section{Compliance with ethical standards}

Guarantor The scientific guarantor of this publication is Peter B. Noël.

Conflict of interest Heiner Daerr, Ewald Roessl and Roland Proksa are employees of Philips Healthcare. The remaining authors (Julia Dangelmaier, Daniel Bar-Ness, Daniela Muenzel, Salim Si-Mohamed, Sebastian Ehn, Alexander A. Fingerle, Melanie A. Kimm, Felix K. Kopp, Loic Boussel, Franz Pfeiffer, Ernst J. Rummeny, Philippe Douek and Peter B. Noël) declare no conflict of interest.

Statistics and biometry No complex statistical methods were necessary for this paper.

Informed consent Written informed consent was not required for this study because the study was phantom-based.

Ethical approval Institutional review board approval was not required because the study was phantom-based.

\section{Methodology \\ - prospective \\ - experimental \\ - performed at one institution}

Open Access This article is distributed under the terms of the Creative Commons Attribution 4.0 International License (http:// creativecommons.org/licenses/by/4.0/), which permits unrestricted use, distribution, and reproduction in any medium, provided you give appropriate credit to the original author(s) and the source, provide a link to the Creative Commons license, and indicate if changes were made.

\section{References}

1. Li X, Zhao G, Zhang J, Duan Z, Xin S (2013) Prevalence and trends of the abdominal aortic aneurysms epidemic in general populationa meta-analysis. PLoS One 8:e81260

2. Norman PE, Jamrozik K, Lawrence-Brown MM et al (2004) Population based randomised controlled trial on impact of mortality from abdominal aortic aneurysm. BMJ 329:1259

3. Forsdahl SH, Singh K, Solberg S, Jacobsen BK (2009) Risk factors for abdominal aortic aneurysms: a 7-year prospective study: the Tromsø Study, 1994-2001. Circulation 119:2202-2208

4. Hirsch AT, Haskal ZJ, Hertzer NR et al (2006) ACC/AHA 2005 Practice Guidelines for the management of patients with peripheral arterial disease (lower extremity, renal, mesenteric, and abdominal aortic): a collaborative report from the American Association for Vascular Surgery/Society for Vascular Surgery, Society for Cardiovascular Angiography and Interventions, Society for Vascular Medicine and Biology, Society of Interventional Radiology, and the ACC/AHA Task Force on Practice Guidelines (Writing Committee to Develop Guidelines for the Management of Patients With Peripheral Arterial Disease): endorsed by the American Association of Cardiovascular and Pulmonary Rehabilitation; National Heart, Lung, and Blood Institute; Society for Vascular Nursing; TransAtlantic Inter-Society Consensus; and Vascular Disease Foundation. Circulation 113:e463-e654

5. Filardo G, Lederle FA, Ballard DJ et al (2013) Immediate open repair vs surveillance in patients with small abdominal aortic aneurysms: survival differences by aneurysm size. Mayo Clin Proc 88: 910-919
6. Parodi JC, Palmaz JC, Barone HD (1991) Transfemoral intraluminal graft implantation for abdominal aortic aneurysms. Ann Vasc Surg 5:491-499

7. Powell JT, Sweeting MJ, Ulug P et al (2017) Meta-analysis of individual-patient data from EVAR-1, DREAM, OVER and ACE trials comparing outcomes of endovascular or open repair for abdominal aortic aneurysm over 5 years. Br J Surg 104:166-178

8. White SB, Stavropoulos SW (2009) Management of endoleaks following endovascular aneurysm repair. Semin Interv Radiol 26: 33-38

9. Chaikof EL, Brewster DC, Dalman RL et al (2009) SVS practice guidelines for the care of patients with an abdominal aortic aneurysm: executive summary. J Vasc Surg 50:880-896

10. Iezzi R, Cotroneo AR, Filippone A et al (2006) Multidetector CT in abdominal aortic aneurysm treated with endovascular repair: are unenhanced and delayed phase enhanced images effective for endoleak detection? Radiology 241:915-921

11. Rozenblit AM, Patlas M, Rosenbaum AT et al (2003) Detection of endoleaks after endovascular repair of abdominal aortic aneurysm: value of unenhanced and delayed helical CT acquisitions. Radiology 227:426-433

12. Brägelmann A, Bunck A, Donas K et al (2013) Dual-energy CT in the follow-up after endovascular abdominal aortic aneurysm repair. RöFo 185:351-357

13. Gutjahr R, Halaweish AF, Yu Z et al (2016) Human imaging with photon counting-based computed tomography at clinical dose levels: contrast-to-noise ratio and cadaver studies. Investig Radiol $51: 421-429$

14. Pourmorteza A, Symons R, Sandfort V et al (2016) Abdominal imaging with contrast-enhanced photon-counting CT: first human experience. Radiology 279:239-245

15. Muenzel D, Bar-Ness D, Roessl E et al (2017) Spectral photoncounting CT: initial experience with dual-contrast agent K-edge colonography. Radiology 283:723-728

16. Si-Mohamed S, Bar-Ness D, Sigovan M et al (2017) Review of an initial experience with an experimental spectral photon-counting computed tomography system. Nucl Instrum Methods Phys Res A $873: 27-35$

17. Cormode D, Si-Mohamed S, Bar-Ness D et al (2017) Multicolor spectral photon-counting computed tomography: in vivo dual contrast imaging with a high count rate scanner. Sci Rep 7:4784

18. Taguchi K, Iwandczyk JS (2013) Vision 20/20: single photon counting X-ray detectors in medical imaging. Med Phys 40:100901

19. Roessl E, Brendel B, Engel KJ, Schlomka JP, Thran A, Proksa R (2011) Sensitivity of photon-counting based K-edge imaging in Xray computed tomography. IEEE Trans Med Imaging 30:16781690

20. Schlomka J, Roessl E, Dorscheid R et al (2008) Experimental feasibility of multi-energy photon-counting K-edge imaging in preclinical computed tomography. Phys Med Biol 53:4031-4047

21. Steadman R, Herrmann C, Livne A (2017) ChromAIX2: a large area, high count-rate energy-resolving photon counting ASIC for a spectral CT prototype. Nucl Instrum Methods Phys Res A 862:18-24

22. Roessl E, Herrmann C (2009) Cramér-Rao lower bound of basis image noise in multiple-energy x-ray imaging. Phys Med Biol 54: 1307-1318

23. Roessl E, Proksa R (2007) K-edge imaging in x-ray computed tomography using multi-bin photon counting detectors. Phys Med Biol 52:4679-4696

24. Alvarez RE, Macovski A (1976) Energy-selective reconstructions in X-ray computerised tomography. Phys Med Biol 21:733-744

25. Brown KM, Zabic S, Shechter G (2015) Impact of spectral separation in dual-energy $\mathrm{CT}$ with anticorrelated statistical reconstruction. In The 13th Int. Meeting on Fully Three-Dimensional Image Reconstruction in Radiology and Nuclear Medicine, Newport, RI, USA, p 491-4 
26. Kalef-Ezra JA, Karavasilis S, Ziogas D, Dristiliaris D, Michalis LK, Matsagas M (2009) Radiation burden of patients undergoing endovascular abdominal aortic aneurysm repair. J Vasc Surg 49: 283-287

27. Javor D, Wressnegger A, Unterhumer S et al (2017) Endoleak detection using single-acquisition split-bolus dual-energy computer tomography (DECT). Eur Radiol 27:1622-1630

28. Symons R, Krauss B, Sahbaee P et al (2017) Photon-counting CT for simultaneous imaging of multiple contrast agents in the abdomen: an in vivo study. Med Phys 44:5120-5127

29. Kalender WA, Kolditz D, Steiding C et al (2017) Technical feasibility proof for high-resolution low-dose photon-counting CT of the breast. Eur Radiol 27:1081-1086
30. Symons R, Cork TE, Sahbaee P et al (2016) Low-dose lung cancer screening with photon-counting CT: a feasibility study. Phys Med Biol 62:202-213

31. Kanda T, Fukusato T, Matsuda M et al (2015) Gadolinium-based contrast agent accumulates in the brain even in subjects without severe renal dysfunction: evaluation of autopsy brain specimens with inductively coupled plasma mass spectroscopy. Radiology 276:228-232

32. Yeh BM, FitzGerald PF, Edic PM et al (2017) Opportunities for new CT contrast agents to maximize the diagnostic potential of emerging spectral CT technologies. Adv Drug Deliv Rev 113: 201-222 